\title{
Study on Control System of Continuous Wave Mud Pulser
}

\author{
Xianfeng Gong and Kai Chen \\ School of Mechanical Engineering, University of Science and Technology Beijing, Beijing 100083, China.
}

\begin{abstract}
In order to transmit the parameters of Measurement While Drilling (MWD) to the ground rapidly and effectively , a high speed continuous wave mud pulser has been developed.In this paper,the author introduces the overall scheme of the control system,including the position servo control of the Permanent Magnet Synchronous Motor (PMSM) based on Space Vector Pulse Width Modulation (SVPWM)and the control of Differential Binary Phase Shift Keying (DBPSK) signal coding .This system succeeds in controlling the carrier frequency in $24 \mathrm{~Hz}$ and the coding rate at $6 \mathrm{bps}$.It meets the needs of engineering applications.
\end{abstract}

Keywords-continuous wave mud pulser; control system; motor control; DBPSK coding

\section{INTRODUCTION}

The drilling fluid pulse generator is an important part of MWD and Logging While Drilling (LWD) equipment[1].The rate of traditional mud pulse generator is $1-3 \mathrm{bps}$. The requires transmission rate which could fit the current drilling requirements is 4-6bps at least[2]. But the data transfer rate of the continuous wave mud pulse system can be used at 6 bits/s, which can be used to solve the bottleneck problem of the realtime data transmission in MWD.Therefore, the study of continuous wave mud pulse system has a vital significance. To this end, the author put forward a high speed continuous wave mud pulser, which controls the rotational speed of the rotor to modulate the pressure signal based on DBPSK coding.

\section{OverALl SCHEME OF THE CONTROL SYSTEM}

\section{A. Components of the Control System}

The function of the control system is to achieve the DBPSK coding by controlling the rotational speed of the PMSM. The control system consists of the control circuit based on Digital Signal Processing (DSP), the driving circuit of the motor, PMSM, resolver, gear reducer, magnetic locator and rotary valve.

\section{B. The Working Principle of the Control System}

PMSM realizes DBPSK coding modulation via control circuit under the control of DSP.This control system uses closed-loop negative feedback control, and the feedback signal is achieved by resolver. In order to monitor the rotary speed of the motor, the resolver position monitoring device is been installed in the tail of the motor, which is coaxial with the motor. The resolver converts the angular position signal of the motor rotor into an analog orthogonal sinusoidal and cosine signal, and the analog orthogonal signal is converted by the resolver converter to a digital position signal which can be recognized by DSP. DSP converts the actual position signal which is outputted by the resolver converter into a speed signal and compares it with the previous setting speed to calculate the error. The error is converted to the Pulse Width Modulation (PWM) pulse via adaptive PID algorithm. And then PWM pulse achieves the speed control of the motor.

\section{THE DESIGN OF THE CONTROL SYSTEM}

The design of the control system mainly includes hardware design and software design.

\section{A. Hardware Design}

According to the function modules, the hardware of the pulser control system can be classified as: power supply module, motor control module, motor drive module, monitoring module and generator control module. These modules are not to do detailed introduction in this paper, and eight circuit boards of these modules are distributed in the triangular electronic skeleton.

\section{B. Software Design}

The function of the software design is to achieve the speed control and coding of the motor. The main modules are PMSM control module and DBPSK coding module.

\section{1) PMSM control module}

The function of the motor control module is to realize the position servo control of the PMSM based on SVPWM.The block diagram of the motor control system is shown in Fig.I.

The speed setting value $\omega r^{*}$ is compared with the actual speed value $\omega r$, and the quadrature-axis current setting value $\mathrm{iq}^{*}$ is generated by the speed regulator. Then $\mathrm{iq}^{*}$ is compared with the actual quadrature-axis current iq to generate the quadrature-axis current error $\Delta \mathrm{q}$.The direct-axis current setting value $\mathrm{id}^{*}$ is compared with the actual current value id to generate the direct-axis current error $\Delta \mathrm{d}$. The direct-axis and the quadrature-axis current errors are converted to the directaxis voltage $\mathrm{Ud}^{*}$ and the quadrature-axis voltage $\mathrm{Uq}^{*}$ by the urrent regulator. Then the $\mathrm{Ud}^{*}$ and $\mathrm{Uq}^{*}$ are converted to $\mathrm{U}^{*}$, $\mathrm{U} \beta^{*}$ in the $\alpha \beta$ coordinate system by Park inverse transformation. The $\mathrm{U} \alpha^{*}$ and $\mathrm{U} \beta^{*}$ control the duty cycle output of inverter by the SVPWM, and the fluctuation of the inverter power supply is considered in SVPWM. In the control system, the actual direct-axis and quadrature-axis currents are calculated by the monitoring current of the motor phase $\mathrm{A}$ and B. A, B and C phase currents are obtained by monitoring the phase current of the motor through the current sensor. Three phase currents are converted to $i \alpha$ and i $\beta$ in the $\alpha \beta$ coordinate system through Clarke transmission, and then converted to the actual current id and iq in the direct-quadrature coordinate 
system through Park transmission. In the process of Park transmission and Park inverse transmission, the position information of the rotor is required, which is detected by resolver installed on the rotor.In addition, the speed of rotor can be obtained by taking a derivative with the data of position.

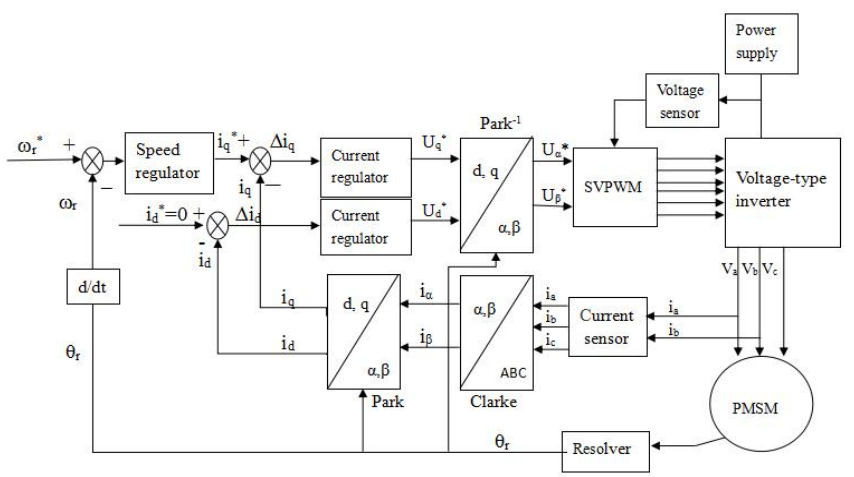

FIGURE I. THE BLOCK DIAGRAM OF THE MOTOR CONTROL SYSTEM

The speed regulators and current regulators are actually Proportion Integration Differentiation (PID) controllers. When a large signal input or other condition make the control system go into a saturated state, the system's performance will have a great reduction, so the system can't meet the requirements. Therefore, control system uses PID control algorithm based on Anti-Windup technology. Control system can still achieve satisfactory performance when the system is in the saturate state by adding the appropriate compensation.

\section{2)DBPSK coding module}

There are only phase " 0 " and phase " $\pi$ " in the phase modulation signal, which represent " 0 " and " 1 ", and the phase of the latter data takes the phase of the previous data as a reference.

Transmission ratio of the pulser system can be calculated. The motor rotates one revolution each time, the magnetic position rotates $1 / 8$ revolution through gear reducer. Because the rotor of the motor has 4 blades, the rotor produces $1 / 2$ sine wave and resolver which is coaxial with the motor produces 4096 counts. When the motor rotates two revolutions each time, the rotor produces a sine wave and resolver produces 8196 counts. The count value can be achieved by a 13-bit counter. When the output of the resolver is 0 , the rotary valve is full open. When the output is 4096, the rotary valve is full closed. Certainly, the initial count can be achieved by the compensation of the initial program.

Continuous wave mud pulser make 4 carrier cycle an a bit when it is in the DBPSK modulation, and frequency of the selected carrier is $24 \mathrm{~Hz}$. When the transmission data is " 0 ", the speed of the motor is constant at 2880RPM. So, as long as the speed of the motor is constant at 2880RPM, the speed of the rotary valve is constant at 360RPM, and the output of mud pressure wave is data " 0 ". But when the output data is " 1 ", a phase lag of $180^{\circ}$ is required, and change of phase is achieved by adjusting the speed of the motor within $60 \mathrm{~ms}$. The phase of the pressure wave is corresponding to the output of the resolver, so the speed of the motor is controlled to make the output of the resolver reduce 4096 counts, and the speed of the rotary valve returns to the normal speed state after the phase change.

The change of phase is achieved by controlling the speed of the motor, and the trapezoid adjustment method is applied in this paper.

The speed of the motor which is outputted by resolver is $196 \mathrm{c} / \mathrm{ms}$ when the $24 \mathrm{~Hz}$ carrier is generated without generating a phase change.

$$
\text { posn_inc }=8192 \times 24 / 1000=196 c / m s
$$
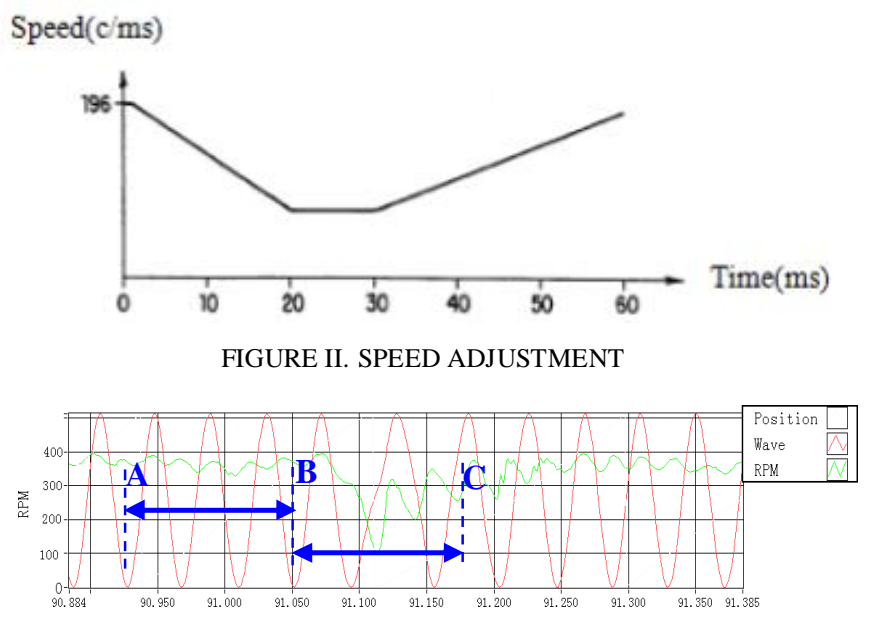

FIGURE III. THE SPEED CONTROL PROCESS OF ROTARY VALVE AT A PHASE LAG OF $180^{\circ}$

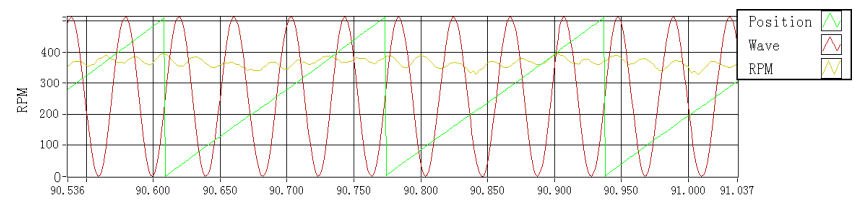

FIGURE IV. THE WAVEFORM AT CODE “0”

Fig.II describes the final velocity synthesis curve. The area surrounded by the trapezoidal curve is 4096 counts, and the speed change curve is selected the trapezoidal method. In the phase shift stage, the speed slows down from $196 \mathrm{c} / \mathrm{ms}$ to 74 $\mathrm{c} / \mathrm{ms}$ with $21 \mathrm{~ms}$, and it is stable at $74 \mathrm{c} / \mathrm{ms}$ with $8 \mathrm{~ms}$, then accelerates to $196 \mathrm{c} / \mathrm{ms}$ with $31 \mathrm{~ms}$.

Fig.III describes the speed control process of rotary valve when a phase lag of $180^{\circ}$ is produced by achieving the code 1.The green curve represents the speed adjustment process of the rotor, and the red curve represents the position of the rotor which is the pressure wave signal. The rotor starts to slow down at $91.09 \mathrm{~s}$, and at near $91.15 \mathrm{~s}$ the speed control is completed. In the Fig.III, $\mathrm{AB}$ and $\mathrm{BC}$ are time periods of the equal length. At point $\mathrm{B}$, the pressure wave is at the bottom, and after the speed control, the pressure wave at point $\mathrm{C}$ is at the peak.

Fig.IV describes the waveform when the code is " 0 ". The yellow curve represents the rotational speed of the rotor. The red curve represents the position curve of the rotor or the mud pressure wave, and the green curve represents the output data 
of resolver. When the code is " 0 ", the speed of rotor is maintained at 360RPM, and the position of resolver has a linear change from 0 to 4095 . When the rotor has a full turn, the position of resolver changes from 4095 to 0 . The mud pressure wave changes in a continuous sinusoidal manner.

\section{THE CODING AND DECODING EXPERIMENTS}

Fig. $\mathrm{V}$ is the principle diagram of signal generator simulation system.

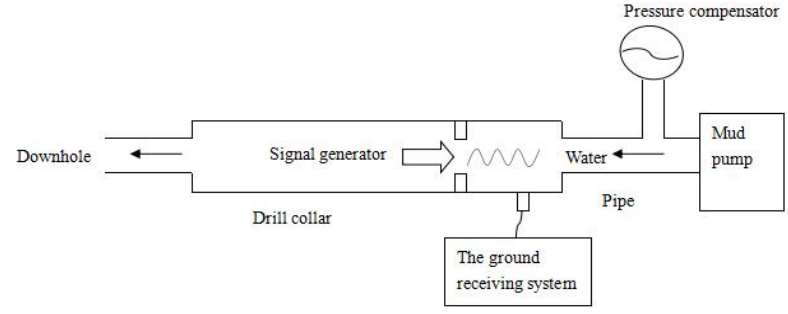

FIGURE V. THE PRINCIPLE DIAGRAM OF SIGNAL GENERATOR SIMULATION SYSTEM

The experimental system uses water instead of mud medium. The system consists of plunger pump, pipe, drill collar, continuous wave pulser and the receiving system on ground.

The parameters of plunger pump is as follows. First, the size is $5 \mathrm{ZB}-108 / 5$.Second, the stoke is $127 \mathrm{~mm}$.Third, the preset pressure is $5 \mathrm{MPa}$.

The parameters of pump is as follows. First, the material is Q235. Second, the inner diameter is $3.1 \mathrm{in}$, and the outer diameter is $3.5 \mathrm{in}$. Third, the length is $100 \mathrm{~m}$.

The experiment on signal generator is used to test the signal strength of the signal generator and the decoding capability of the receiving system on the ground under the different pressure and flow conditions. The experiments are conducted when the system pressure is $0.75 \mathrm{MPa}$ and the flow is $3 \mathrm{~L} / \mathrm{m}$.
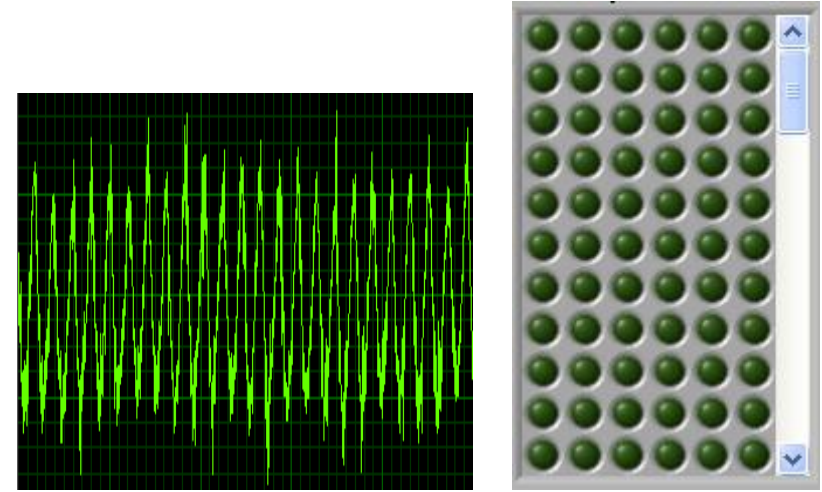

FIGURE VI. THE WAVEFORM AT CODE "0" AND DECODING “0”

In the Figure VI, the first figure describes the waveform which is received by the ground system when the signal generator send all " 0 " and the second figure describes the decoding result. So when the code is " 0 ", the decoding rate is $100 \%$.
In the Figure VII, the first figure describes the waveform which is received by the ground system when the signal generator send all "1" and the second figure describes the decoding result, and the decoding rate is $92 \%$.
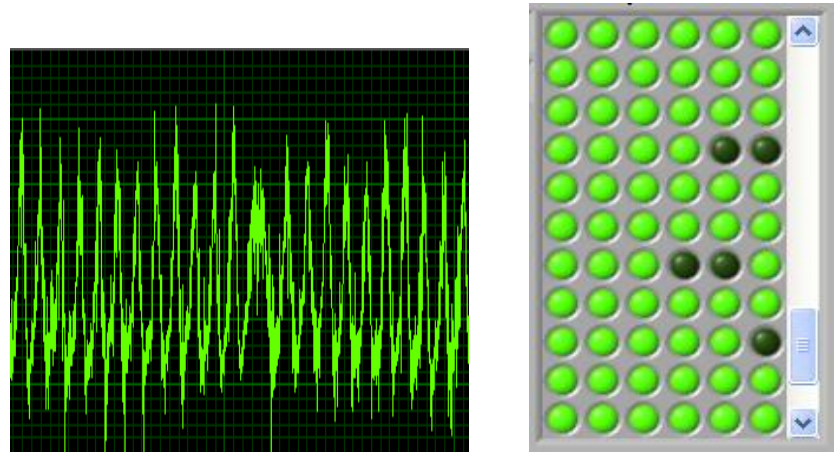

FIGURE VII. THE WAVEFORM AT CODE"1" AND DECODING “1”

\section{CONCLUSION}

In this paper, we design the software and program, and we do some experiments. We get the following results. First, this paper achieves the design of the continuous wave pressure signal generator. It succeeds in controlling the frequency of carrier in $24 \mathrm{~Hz}$ and the coding rate at 6bps. Second, the control based on SVPWM of PMSM is achieved by DSP, so the motor is more reliable and the speed control is more stable. It can achieve $62 \%$ speed range with $20 \mathrm{~ms}$, and it can meet the coding requirements. Third, the system achieves the code of pulser by using DBPSK coding, and it can achieve all "0" code, all " 1 " code, periodic "00001" code and so on. Thus, this control system can be applied in engineering applications.

\section{ACKNOWLEDGMENT}

The authors want to thank anonymous referees for their valuable comments that led to an improvement in this paper.

\section{REFERENCES}

[1] Hutin R, Tennent W, Kashikar S V. New mud pulse telemetry techniques for deepwater applications and improved Real-time data capabilities . In proceeding of the SPE/IADC 67762,2001.

[2] Jing Shao, Zhidan Yan,Suli Han, Hui Li, Tingzheng Gao,Xiufeng Hu, Chunming Wei. Differential signal extraction for continuous wave mud pulse telemetry[J]. Journal of Petroleum Science and Engineering,2016,pp.127-130.

[3] Spinner T G, Stone F A. Mud Pulse Logging While Drilling System Design, Development, and Demonstration, IADC/Caodc Drill. Tech. Conf., Houston, Tex, 1978.

[4] Franceo Donat, Joachim Oppelt, A Trampini, Detlef Rognitz,. Innovative Rotary Closed Loop System.SPE 39328,1998.

[5] Heisig G.,Sancho J. Downhole diagnosis of drilling dynamics data provides new level drilling process[J].JPT,1999,pp.38-39.

[6] Xingliu Hu,Dehua Liu.Research and application of SVPWM technique in inverter.Telecom Power Technologies,2004,pp.12-15.

[7] Underwood L.D., Odell C. A System Approch to Downhole Adjustable Stabilizer Design and Application. SPE 27484.

[8] Jinsong Zhang,Hongmei Ma. The software realization of digital modulation and demodulation. Radio Communication Technology,2002,pp.31-33.

[9] Patton B.J, Gravley W, Godbey J.K, Sexton, J. H., Hawk, D. E., Slover V. R., Harrell J.W. Development and Successful Testing of a Continuous-Wave, Logging-While-Drilling Telemetry System[J]. Journal of Petroleum Technology, 1981. 